\title{
Produção, comercialização e exportação de produtos apícolas: uma análise do desempenho da região nordeste brasileira
}

Production, marketing and export of bee products: an analysis of the performance of the brazilian northeast region

Producción, comercialización y exportación de productos apícolas: un análisis del desempeño de la región nororiental brasileña

Michael Douglas Sousa Leite ORCID: https://orcid.org/0000-0002-9356-1872

Faculdade Católica Santa Teresinha, Brasil

E-mail: michaeldouglas_adm@hotmail.com

Aline Carla de Medeiros

ORCID: https://orcid.org/0000-0002-0161-3541

Universidade Federal de Campina Grande, Brasil

E-mail: alinecarla.edu@gmail.com

Patrício Borges Maracajá

ORCID: https://orcid.org/0000-0003-4812-0389

Universidade Federal de Campina Grande, Brasil E-mail: patricio@ufcg.edu.br

Kévia Katiúcia Santos Bezerra

ORCID: https://orcid.org/0000-0002-2310-0034 Universidade Federal de Campina Grande, Brasil

E-mail: keviabezerra@gmail.com

Mateus Gonçalves Silva

ORCID: https://orcid.org/0000-0003-0412-6359 Universidade Federal de Campina Grande, Brasil

E-mail: matheus.goncalves2102@gmail.com

Sandra Maijane Soares de Belchior

ORCID: https://orcid.org/0000-0001-5807-2259

Faculdades Integradas do Ceará, Brasil E-mail: sandrabelchior@hotmail.com

Helmo Robério Ferreira de Meneses

ORCID: https://orcid.org/0000-0001-9326-8548 Faculdades Integradas do Ceará, Brasil

E-mail: helmo_rob@hotmail.com

Glauber Iure Cardoso de Menezes Silva

ORCID: https://orcid.org/0000-0002-8483-4488 Faculdades Integradas do Ceará, Brasil E-mail: glauber.adv@bol.com.br

Thaise de Abreu Brasileiro Sarmento

ORCID: https://orcid.org/0000-0003-0390-805X

Universidade Federal de Campina Grande, Brasil E-mail: thaiseabreu@hotmail.com

Mariana Ferreira Pessoa

ORCID: https://orcid.org/0000-0002-1563-5469 Universidade do Estado do Rio Grande do Norte, Brasil E-mail:marianafepessoa@gmail.com

José Carlos Gomes

ORCID: https://orcid.org/0000-0001-9361-8595 Instituto Federal da Paraíba, Brasil E-mail: zc.gomesagro@gmail.com

Pablo Sthefano Roque de Souza Bandeira ORCID: https://orcid.org/0000-0002-8980-5977 Universidade Regional do Cariri, Brasil E-mail: bandeiraroque@gmail.com

Daniel Souza Cesar

ORCID: https://orcid.org/0000-0003-1983-8818 Faculdades Integradas do Ceará, Brasil E-mail: prof.danielcesar@gmail.com 


\author{
Aline Cristina de Araújo Florentino Silva \\ ORCID: https://orcid.org/0000-0002-0091-6946 \\ Faculdades Integradas do Ceará, Brasil \\ E-mail: alineflorentino.fasp@gmail.com \\ Ana Karine Gomes de Figueiredo \\ ORCID: https://orcid.org/0000-0002-4305-0847 \\ Universidade Federal de Campina Grande, Brasil \\ E-mail: Ana.kgfigueiredo@gmail.com \\ Kadydja Mayara Ramos Nobre \\ ORCID: https://orcid.org/0000-0003-2269-1220 \\ Universidade Federal de Campina Grande, Brasil \\ E-mail: kadydja_mrn@hotmail.com \\ Sauly Martinho Gomes de Sousa \\ ORCID: https://orcid.org/0000-0001-6181-0363 \\ Faculdades Integradas do Ceará, Brasil \\ E-mail: administracao@sousa.pb.gov.br \\ Tiago Douglas Cavalcante Carneiro \\ ORCID: https://orcid.org/0000-0003-1183-1718 \\ Faculdade Católica Santa Teresinha, Brasil \\ E-mail: tiagocarneirocaico@gmail.com \\ Albimah Medeiros de Araujo \\ ORCID: https://orcid.org/0000-0002-8251-0869 \\ Universidade Federal de Campina Grande, Brasil \\ E-mail: albimahmedeiros64@gmail.com \\ Júlia Marcia Lourenço de Almeida Martins Medeiros \\ ORCID: https://orcid.org/0000-0001-8554-8136 \\ Universidade Federal de Campina Grande, Brasil \\ E-mail: juliaalmeidamartins@gmail.com
}

\begin{abstract}
Resumo
A apicultura brasileira vem ganhando destaque desde a introdução das abelhas Apís mellifera provenientes da África, processo este que acarretou a hibridização das espécies europeias já existentes no país. O cruzamento entre essas espécies de abelhas promoveu ampla disseminação de subespécies por todas as regiões do Brasil, as quais foram se adaptando as adversidades, se tornando resistentes e mais produtivas. Na região Nordeste, as subespécies de abelhas resultantes da hibridização, abelhas africanizadas, se adaptaram muito bem às condições de clima quente e vegetação de Caatinga, passando a promover às famílias de agricultores renda extra pela prática da apicultura racional. Nesse contexto, o artigo se propõe a realizar um levantamento do desempenho da região nordeste brasileira em relação a produção, comercialização e a exportação de produtos apícolas. Para tanto utilizou-se de uma pesquisa bibliográfica e documental realizada a partir das bases de dados da Biblioteca Científica Eletrônica em Linha (Scielo) e Portal de Periódicos CAPES/MEC. Com base na análise dos dados obtidos, evidenciou-se que a região Nordeste do Brasil possui destaque na produção, comercialização e exportação de produtos apícolas, com realce para o mel como o principal produto, ficando atrás somente das regiões Sul e Sudeste. O mel do Nordeste apresenta qualidade imensurável pelo baixo índice de contaminação de resíduos químicos, o que permite alto poder de competitividade internacional. Para um crescimento constante da apicultura no Nordeste, é necessário o investimento em pesquisas e desenvolvimento de tecnologias para o manejo agroindustrial da cadeia apícola.
\end{abstract}

Palavras-chave: Apicultura; Apis melifera; Mercado; Produtos das abelhas.

\begin{abstract}
Brazilian beekeeping has gained prominence since the introduction of the Apís mellifera bees from Africa, a process that led to the hybridization of European species already existing in the country. The crossing between these species of bees promoted a wide spread of subspecies throughout all regions of Brazil, which were adapting to adversities, becoming resistant and more productive. In the Northeast region, the subspecies of bees resulting from hybridization, Africanized bees, adapted very well to the conditions of hot climate and Caatinga vegetation, starting to promote extra income to farming families through the practice of rational beekeeping. In this context, the article proposes to carry out a survey of the performance of the Brazilian northeast region in relation to the production, commercialization and export of bee products. For that, a bibliographic and documentary research was used, carried out from the databases of the Online Electronic Scientific Library (Scielo) and CAPES/MEC Journal Portal. Based on the analysis of the data obtained, it was evident that the Northeast region of Brazil stands out in the production, marketing and export of bee products, with honey as the main product, only behind the South and Southeast regions. Honey from the Northeast presents immeasurable quality due to the low level of chemical residue contamination, which allows for a high level of international competitiveness. For a constant growth of beekeeping in the Northeast, investment in research and development of technologies for the agro-industrial management of the beekeeping chain is necessary.
\end{abstract}

Keywords: Beekeeping; Apis mellifera; Marketplace; Bee products. 


\begin{abstract}
Resumen
La apicultura brasileña ha ganado protagonismo desde la introducción de las abejas Apís mellifera de África, proceso que condujo a la hibridación de especies europeas ya existentes en el país. El cruce entre estas especies de abejas promovió una amplia difusión de subespecies en todas las regiones de Brasil, que se fueron adaptando a las adversidades, volviéndose resistentes y más productivas. En la región Nordeste, las subespecies de abejas resultantes de la hibridación, las abejas africanizadas, se adaptaron muy bien a las condiciones de clima cálido y vegetación de Caatinga, comenzando a promover ingresos extra a las familias campesinas a través de la práctica de la apicultura racional. En este contexto, el artículo propone realizar un relevamiento del desempeño de la región nororiental brasileña en relación a la producción, comercialización y exportación de productos apícolas. Para ello, se utilizó una investigación bibliográfica y documental, realizada a partir de las bases de datos de la Biblioteca Científica Electrónica en Línea (Scielo) y el Portal de Revistas CAPES / MEC. A partir del análisis de los datos obtenidos, fue evidente que la región Nordeste de Brasil se destaca en la producción, comercialización y exportación de productos apícolas, con la miel como principal producto, solo por detrás de las regiones Sur y Sudeste. La miel del Nordeste tiene una calidad inconmensurable debido al bajo nivel de contaminación de residuos químicos, lo que permite un alto nivel de competitividad internacional. Para un crecimiento constante de la apicultura en el Nordeste, es necesaria la inversión en investigación y desarrollo de tecnologías para el manejo agroindustrial de la cadena apícola.
\end{abstract}

Palabras clave: Apicultura; Apis mellifera; Mercado; Productos apícolas.

\title{
1. Introdução
}

O interesse pela apicultura vem do ser humano desde o início das civilizações antigas, fornecendo fontes ricas de alimentos e produtos terapêuticos. Os achados mais antigo de aproveitamento dos produtos apícolas é de pinturas rupestres com mais de 7.000 anos a.C., encontrado em Valencia na Espanha (Silva, 2014). Nessas pinturas rupestres se observam figuras de homens coletando favos de mel em cavidades rochosas com abelhas voando ao redor (Silva et al., 2018).

A apicultura se caracteriza pela exploração racional e econômica das abelhas do gênero Apis e da espécie Apis mellífera, sendo sua introdução no Brasil datada desde 1839 (Scaramucci \& Silva, 2014). Contudo é apenas praticada com maior intensidade a partir da imigração dos europeus (alemães e italianos) para o Brasil, que, no início do século XIX, trouxeram as abelhas europeias (Silva et al., 2020).

É uma atividade de relevância reconhecida na geração de renda e emprego, fator de diversificação da propriedade rural, proporcionando diversos benefícios sociais, ecológicos e econômicos (Souza et al., 2012). Por todo o país, são desenvolvidas as atividades apícolas, gerando milhares de empregos diretos e indiretos, apenas na confecção de equipamentos, no manejo de diversos produtos de mel, nos empregos de manutenção dos apiários, da venda da cera, pólen, apitoxina, geleia real, polinização de pomares, da flora silvestre, cultivos agrícolas e dentre outros (Vidal, 2019).

Segundo dados da Organização das Nações Unidas para Alimentação e Agricultura - FAO (2019), no Brasil, no ano de 2017 a produção do mel chegou a uma estimativa de 20 a 49 mil toneladas, produção semelhante a países como Uruguai, Espanha, Canadá e Alemanha. A região Nordeste, por exemplo, apresenta índices altos de competitividade no mercado exterior devido a qualidade do mel produzido na região (Aires-Neto \& Carvalho, 2017; Vidal, 2019; Silva et al., 2020).

Contudo, os dados nacionais mostram que um dos entraves para a expansão da produção de produtos apícolas no Brasil é o baixo consumo, em especial do Mel, que está conexo ao baixo nível de renda e também a falta de hábito de consumo, além da visão de que o mel é um produto apenas de uso medicinal, desconhecendo suas características nutricionais (Paula et al., 2016).

De acordo com o Serviço Brasileiro de Apoio às Micro e Pequenas Empresas - SEBRAE (2009) o consumo de mel no Brasil é um dos mais baixos do mundo, não ultrapassando 120 gramas/ano por habitante, índices estes, bem diferente dos 3,4 kg/ano da República Centro-Africana; 1,8 kg/ano da Nova Zelândia; 1,7 kg/ano da Angola; 1,5 kg/ano da Grécia; 1,4 kg/ano da Áustria; 1,3 kg/ano da Suíça; 1,2 kg/ano da União Europeia; 1,1 kg/ano da Alemanha, 847 gramas/ano da Espanha, 576 gramas/ano dos Estados Unidos e 310 gramas/ano do México (Paula et al., 2016).

Em relação a produção, apesar do grande potencial brasileiro para a produção apícola e de ter excelente reputação com relação à qualidade do produto exportado, o país responde por apenas 4,0\% das exportações do produto no mundo. No entanto, 
o Brasil é o maior fornecedor de mel orgânico para os Estados Unidos, segundo dados do United States Department of Agriculture - USDA (2019), mais de 90\% de todo o mel orgânico importado pelos EUA foi procedente do Brasil, sendo o nosso mel bastante valorizado no mercado americano (Vidal, 2019).

Nesse sentido o presente artigo busca realizar uma análise do desempenho da região nordeste brasileira em relação a produção, comercialização e a exportação de produtos apícolas.

\section{Metodologia}

A pesquisa em tela, configura-se como do tipo bibliográfica de natureza qualitativa e fundamentada, para tanto, se utilizou de uma análise documental. A natureza bibliográfica segundo Marconi \& Lakatos (2017) baseia-se em material já elaborado constituindo-se principalmente de livros, artigos, dissertações e teses. Gil (2019), acrescentando-se o fato de que pesquisa alguma parte atualmente da estaca zero e em algum lugar, pesquisas semelhantes já podem ter sigo feitas ou apenas complementadas.

O método qualitativo caracteriza-se por sua vez, pela a complexidade de determinado problema, sendo necessário compreender e classificar os processos dinâmicos vividos nos grupos, contribuir no processo de mudança, possibilitando o entendimento mais claro do assunto (Marconi \& Lakatos, 2017).

Para levantamento dos artigos foram utilizadas duas bases: Biblioteca Científica Eletrônica em Linha (Scielo) e Portal de Periódicos CAPES/MEC e os descritores "Apicultura", "Mercado" e "Nordeste". A princípio, a busca pelos descritores foi dada individualmente, utilizando-se posteriormente o cruzamento a partir do operador booleano "and". Desse modo, para a seleção da amostra, foram válidos os seguintes critérios: artigos publicados apenas no idioma português, no período de 2010 a 2021, que retrataram a temática em estudo. Sendo assim, foram excluídos aqueles que fugiam da faixa de tempo e com idioma divergente.

Além disso, foram usados sites como: IBGE, SENAR, SEBRAE, FAO, onde coletou os dados sobre a produção, comercialização e exportação de produtos apícolas na região nordeste.

\section{A Produção de Produtos Apícolas no Nordeste}

A apicultura brasileira é predominantemente de base familiar, onde pequenos apiários no geral são mantidos por famílias de agricultores com base de produção agroecológica e inseridos em dinâmicas da economia solidária (Liberato \& Morais, 2016). Uma das características básicas dessa atividade é a pouca exigência em mão-de-obra e recursos. Desta maneira, a apicultura vem sendo desenvolvida como uma atividade produtiva que traz possibilidades de uma renda adicional e que favorece aos agricultores um consumo mais frequente do mel na dieta, sem falar nos diversos benefícios da polinização das plantas frutíferas, nativas e exóticas que induz a ampliação do volume de sementes e frutos, e consequentemente o crescimento da cobertura vegetal e maior produtividade (Paula et al., 2016).

Como já foi mencionado a federação brasileira possui a maior capacidade de produção do mundo de mel orgânico, o Nordeste em particular, tem uma boa competitividade no mercado de produtos apícolas (Vidal, 2020). O diferencial do mel da região Nordeste está na contaminação baixa por resíduos de antibióticos e pesticidas, pois boa parte do mel produzido nessa região é proveniente de vegetação nativa. Além disso, a relativa baixa umidade do ar impede o adoecimento das abelhas, dispensando a utilização de medicamentos (Liberato \& Morais, 2016; Barbosa \& Pereira, 2019).

Na Tabela 1 abaixo é destacado o número de estabelecimentos agropecuários com apicultura de acordo com último censo agropecuário de 2017 no Brasil. 
Tabela 1 - Número de estabelecimentos agropecuários com apicultura por Região. BRASIL-2017.

\begin{tabular}{lc}
\hline \multicolumn{1}{c}{ Grande Região } & Número de estabelecimentos \\
\hline Norte & 2154 \\
Nordeste & 24150 \\
Sudeste & 7049 \\
Sul & 66483 \\
Centro-Oeste & 1961 \\
\hline Total & $\mathbf{1 0 1 7 9 7}$ \\
\hline
\end{tabular}

Fonte: IBGE (2017).

Das cinco regiões brasileiras, o Sul é a região com maior número de estabelecimentos com apicultura, com cerca de 66.483, já a região Nordeste encontra-se em segundo lugar com 24.150 estabelecimentos, e o Sudeste em terceiro com 7.049, nas outras duas regiões o número de estabelecimentos é bem abaixo.

A apicultura tem uma relevante importância social, concentrada na região do semiárido, especificamente nos estados do Ceará, Piauí e Bahia, onde no meio rural são poucas as opções produtivas rentáveis devido às limitações na Região, em especial com relação a questão da escassez de água (Vidal, 2020).

No Gráfico 1 é destacado a produção mundial de mel no período de 2013 a 2020. Vale destacar que a China é o maior exportador do mundo de mel e o principal fornecedor para União Europeia (AGROSTAT, 2021). O mel da China é um dos mais baratos do mercado, o baixo custo produtivo faz um dos méis mais competitivos do mundo. A China comercializa o mel com preços mais baixos a nível mundial, contudo, importa pagando bem mais que media mundial (Vidal, 2019).

Gráfico 1 - Produção mundial de mel entre 2013 a 2020, em mil toneladas.

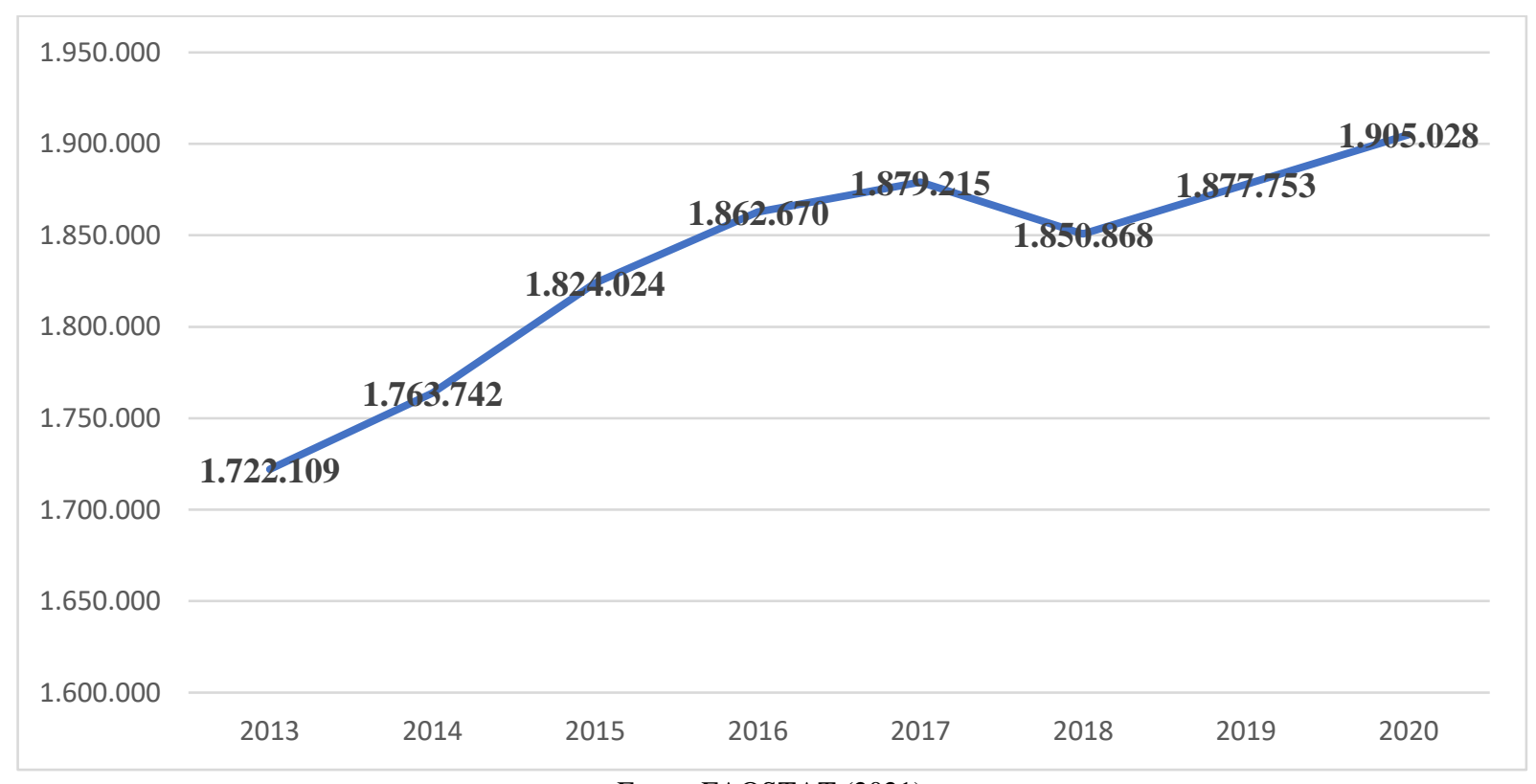

Fonte: FAOSTAT (2021).

Nesse período de 2013 a 2020 a produção de mel mostra um crescimento de mais de 10\%, saindo de uma produção em 2013 de 1.722.109 toneladas para 1.905.028 toneladas. Segundo a FAO (2019) a produção de mel mundial teve uma tendência de aumento nos últimos 20 anos, mesmo com as flutuações nos preços, em países e regiões, adjudicadas a um crescimento na produção por colônia e número de colmeias. O consumo aumentou consideravelmente também nos últimos anos, sendo 
relacionado ao aumento nos padrões de vida e a um maior interesse em produtos saudáveis e naturais (Araújo, Correia \& Silva, 2016).

Em relação a produção brasileira, os últimas 20 anos correspondem ao período em que a produção apícola mais se expandiu, com uma intensidade maior nos últimos 10 anos (Farias et al., 2021). A apicultura apresenta hoje uma posição de grande destaque internacional e nacional pela produção de mel e outros produtos, com características diferentes e excelente qualidade orgânica (Gomes et al., 2017).

O Gráfico 2 mostra o crescimento da produção brasileira de mel entre 2013 e 2020, em toneladas.

Gráfico 2 - Produção brasileira de mel entre 2013 e 2020, em mil toneladas.

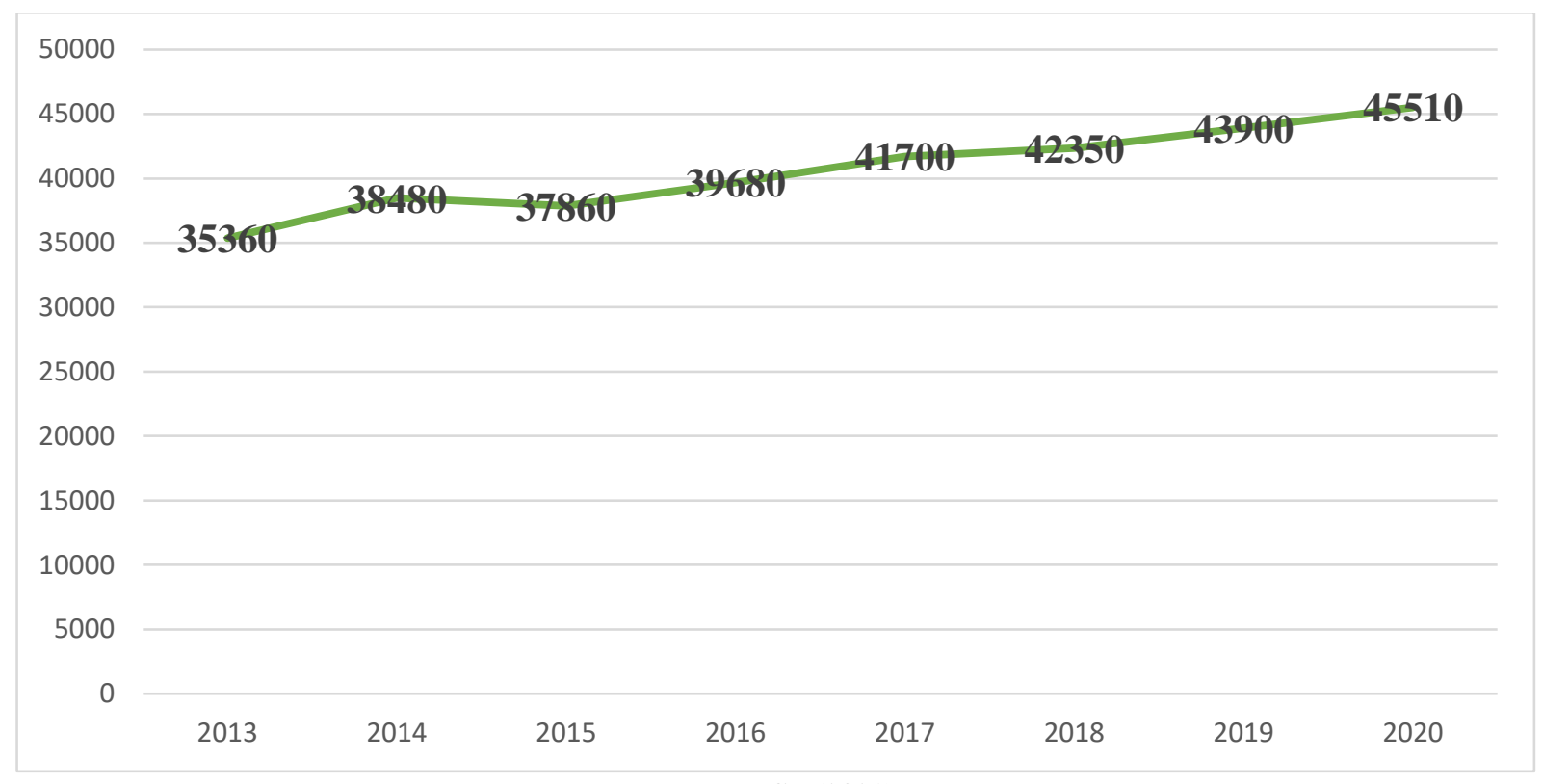

Fonte: IBGE (2021).

No período de 2013 a 2020, houve um crescimento de 28,70\% na produção de mel, um aumento de quase 3 vezes a mais que a produção mundial. Atualmente se estima que a produção de mel nacional possa chegar ao redor das 50 mil toneladas por ano, embora os dados do IBGE não confirmem esses números, uma vez que não existe ainda um levantamento minucioso da produção de mel em todo território nacional (Araujo, Correia \& Silva, 2016). Os únicos dados existentes no Brasil em relação a produção de produtos apícolas são os dados cadastrados no site do IBGE.

Em relação a produção por região no Brasil, o Fráfico 3 mostra as quedas e aumentos na produção em relação aos anos. 
Gráfico 3 - Produção de mel em mil toneladas por região brasileira entre 2011 e 2020.

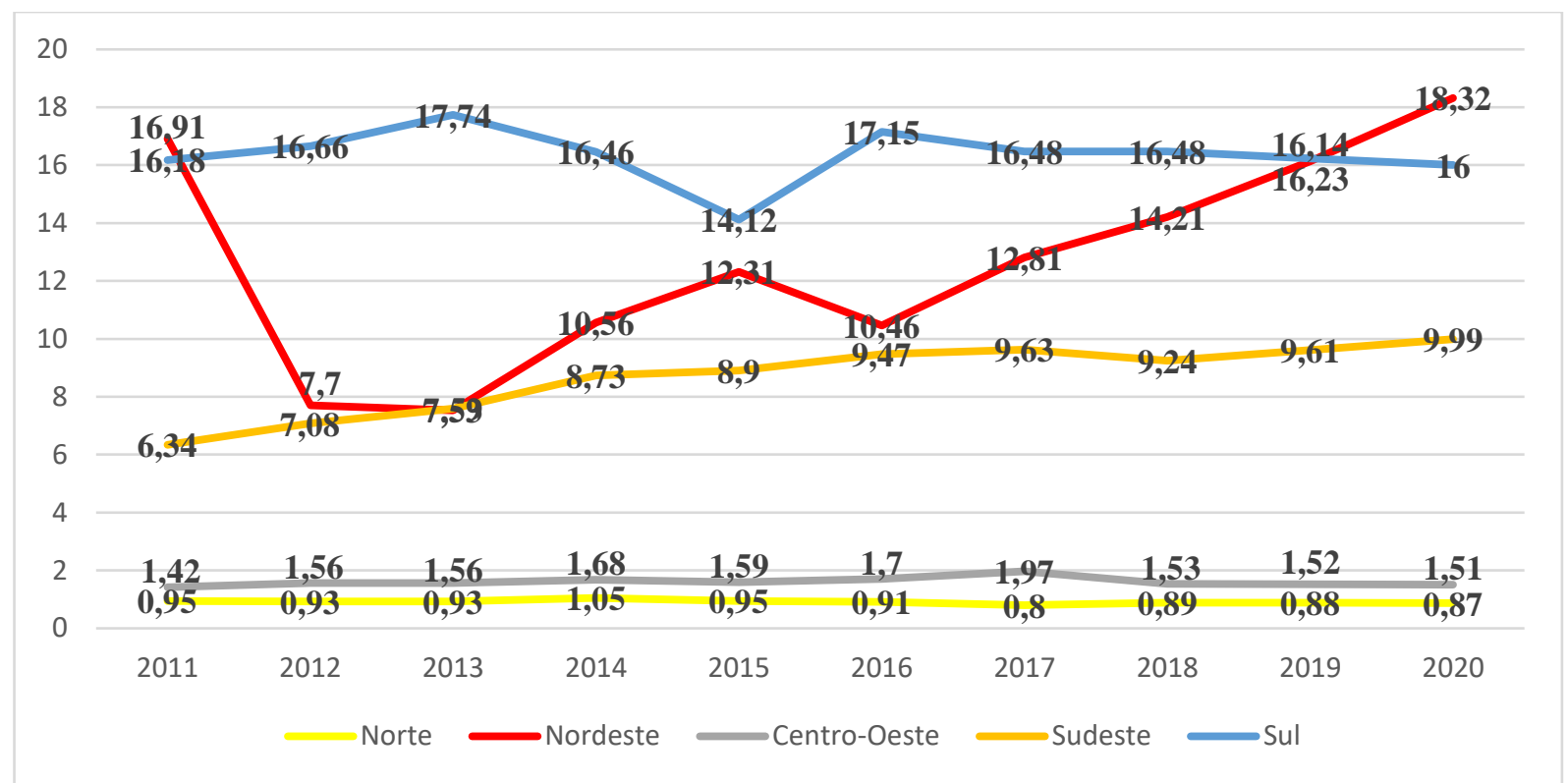

Fonte: IBGE (2021).

Os dados mostram que em números, a região Sul é a que mais produziu nos últimos 10 anos, seguido pela região Nordeste. Em terceiro lugar a região Sudeste mostrou um bom desempenho e as regiões Norte e Centro-Oeste foram as que tiveram menores resultados. Assim, com base nos dados dos últimos anos, o Nordeste mostra um aumento significativo das atividades apícolas e seu enorme potencial de crescimento, até mesmo com a produção de materiais apícolas com qualidade excelente (Souza et al., 2021).

No Gráfico 4 é destacado a produção em mil toneladas por estado da região Nordeste entre 2011 e 2020.

Gráfico 4 - Produção de mel em mil toneladas por estado da região Nordeste do Brasil entre 2011 e 2020.

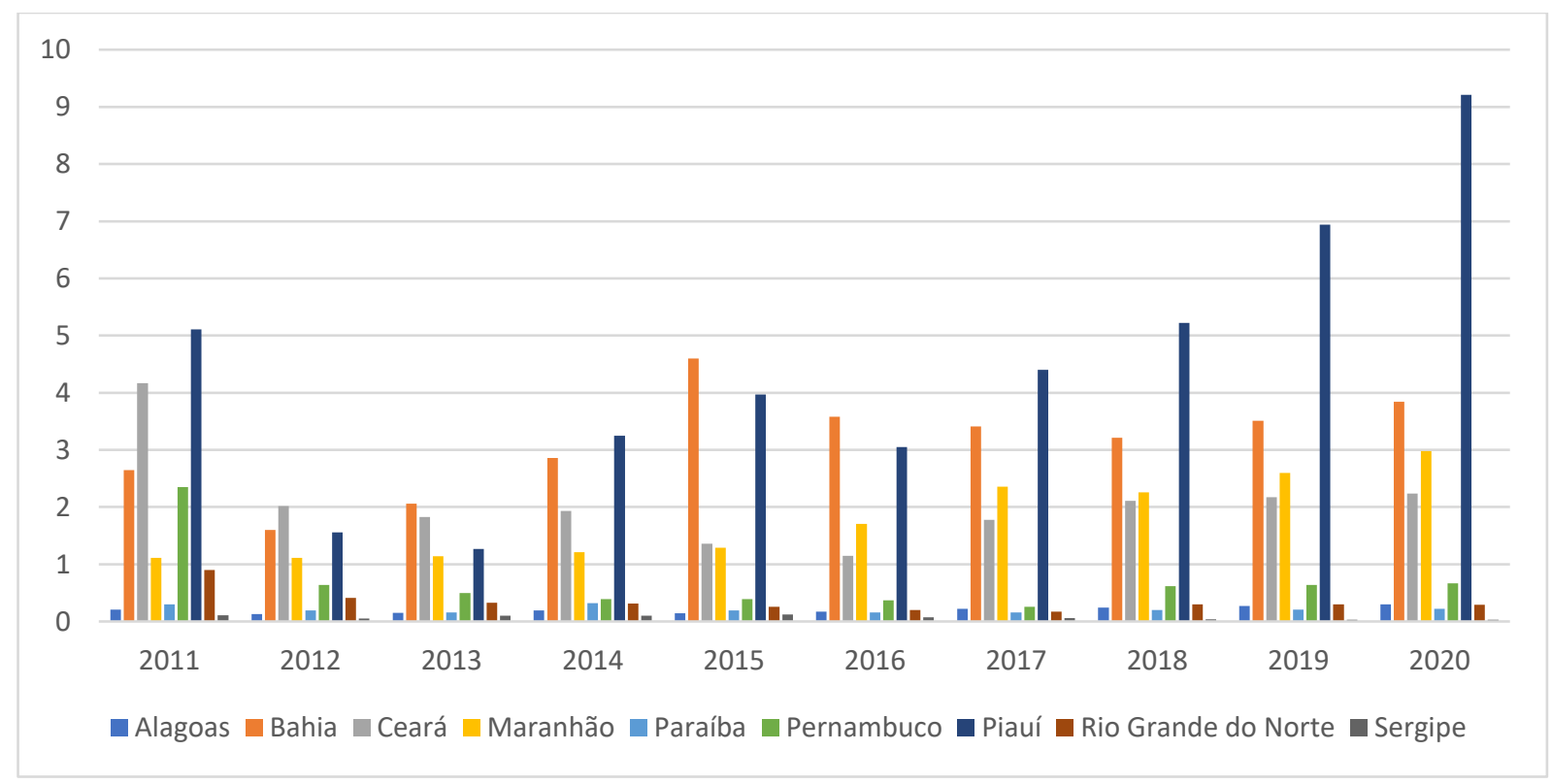

Fonte: IBGE (2021).

Dentre os 09 estados da Região Nordeste, o Piauí, Ceará, Pernambuco, Bahia e Maranhão se destacam como produtores de mel. O Rio Grande do Norte até pouco tempo atrás nem aparecia como produtor de mel no cenário nacional, porém nos 
últimos anos vem aumentando muito a sua produção, e recentemente já começou até exportar o mel produzido (Carvalho et al., 2019).

Embora o setor da apicultura venha se destacando bastante no Nordeste, a região é ainda carente de centros e laboratórios de pesquisas em relação as abelhas e análises de produtos em geral, ficando os apicultores desamparados no apoio para resolver as suas dificuldades vinculadas a produção apícola (Farfan et al., 2020).

No Nordeste os poucos laboratórios de pesquisas são vinculados às Universidades que realizam pesquisas sobre abelhas, como: Universidade Federal de Campina Grande (UFCG), Universidade Federal da Paraíba (UFPB), Universidade Federal do Recôncavo da Bahia (UFRB), Universidade Federal da Bahia (UFBA), Universidade Estadual do Sudoeste da Bahia (UESB), Universidade Estadual de Feira de Santana (UEFS), Universidade Federal do Piauí (UFPI), Universidade Federal do Ceará (UFCE), Universidade Estadual do Maranhão (UEMA), Universidade Federal do Maranhão (UFMA), Universidade Federal de Alagoas (UFAL), Universidade Federal Rural do Semiárido (UFERSA), Universidade Federal do Rio Grande do Norte (UFRN) e algumas instituições estaduais que são vinculadas às Secretarias de Agricultura. No entanto, existe a falta de vínculo das instituições com as federações de Apicultura dos respectivos estados, para um apoio efetivo aos apicultores (Farfan et al., 2020).

Embora a produção apícola nordestina venha crescendo nos últimos anos, significativamente, mostrando ser uma importante área, infelizmente é notório o prejuízo anual dos apicultores frente à elevada taxa de enxameação das abelhas africanizadas (Souza et al, 2021). Este comportamento é uma das características das abelhas africanizadas e que se agrava no Nordeste face às características do clima semiárido que é anualmente castigado pela escassez de chuvas e seca, com consequente falta de água e alimento para estes animais (Liberato, 2016). Paralelamente a esses problemas existem outros certamente, e se constata a elevada agressividade dessas abelhas, chegando a ser quatro vezes maior no Nordeste comparada com a região Sudeste (Pereira et al., 2017).

\section{A Comercialização de Produtos Apícolas}

A comercialização de mel é um contíguo de medidas que permitem colocar esse produto no mercado (SENAR, 2010). $\mathrm{O}$ apicultor pode comercializar o seu mel através da venda direta ao consumidor final, a uma empresa do ramo para o processamento industrial ou por meio de um intermediário. Além disso, o pequeno e médio produtor pode em alguns municípios ter o auxílio das cooperativas que apresentam uma forma importante de comercialização (Cunha, Machado \& Costa, 2014).

O mel pode ser comercializado no mercado internacional e nacional, podendo ser vendido na forma fracionada (em bisnagas, potes ou garrafas de plástico ou vidro) ou em maiores quantidades em tambores ou baldes para indústrias alimentícias, cosméticas e farmacêuticas (Silva, 2010). A comercialização do mel deve seguir as exigências dos Órgãos fiscalizadores: Ministério da Agricultura, Pecuária e Abastecimento - MAPA e Agência Nacional de Vigilância Sanitária - ANVISA (Cunha, Machado \& Costa, 2014). As empresas que fazem esse processamento do mel, compram o mel dos apicultores e fazem a homogeneização, posterior o envase em embalagens de maneira fracionada e apropriadas, e a rotulagem para depois encaminhar o produto para o mercado.

Abaixo destaca-se no Gráfico 5, valores da venda de mel, em reais, por região brasileira. 
Research, Society and Development, v. 10, n. 10, e466101018897, 2021

(CC BY 4.0) | ISSN 2525-3409 | DOI: http://dx.doi.org/10.33448/rsd-v10i10.18897

Gráfico 5 - Valor de venda de mel (em Real) por região brasileira no ano de 2017.

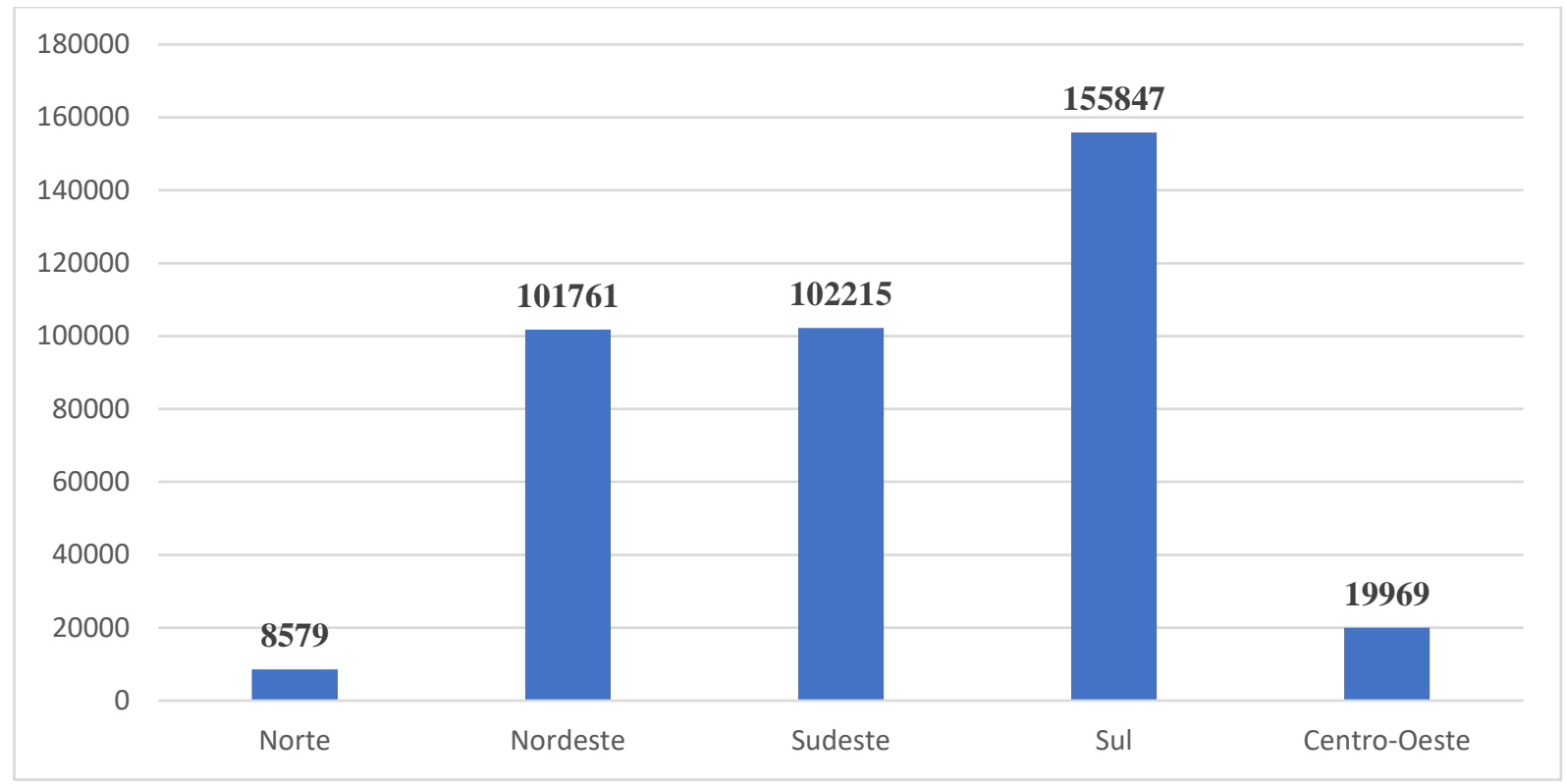

Fonte: IBGE (2017).

A região Sul foi a que mais vendeu mel no ano de 2017, cerca de 155.847 .00 reais, seguido pela região Sudeste e Nordeste. As outras regiões, Centro-Oeste e Norte tiverem desempenho bem abaixo.

No Gráfico 6 são destacados os valores representativos da venda de cera de abelha, geleia real e própolis por região brasileira.

Gráfico 6 - Valor de venda de Cera de abelha, geleia real e própolis, em reais, por região no ano de 2017.

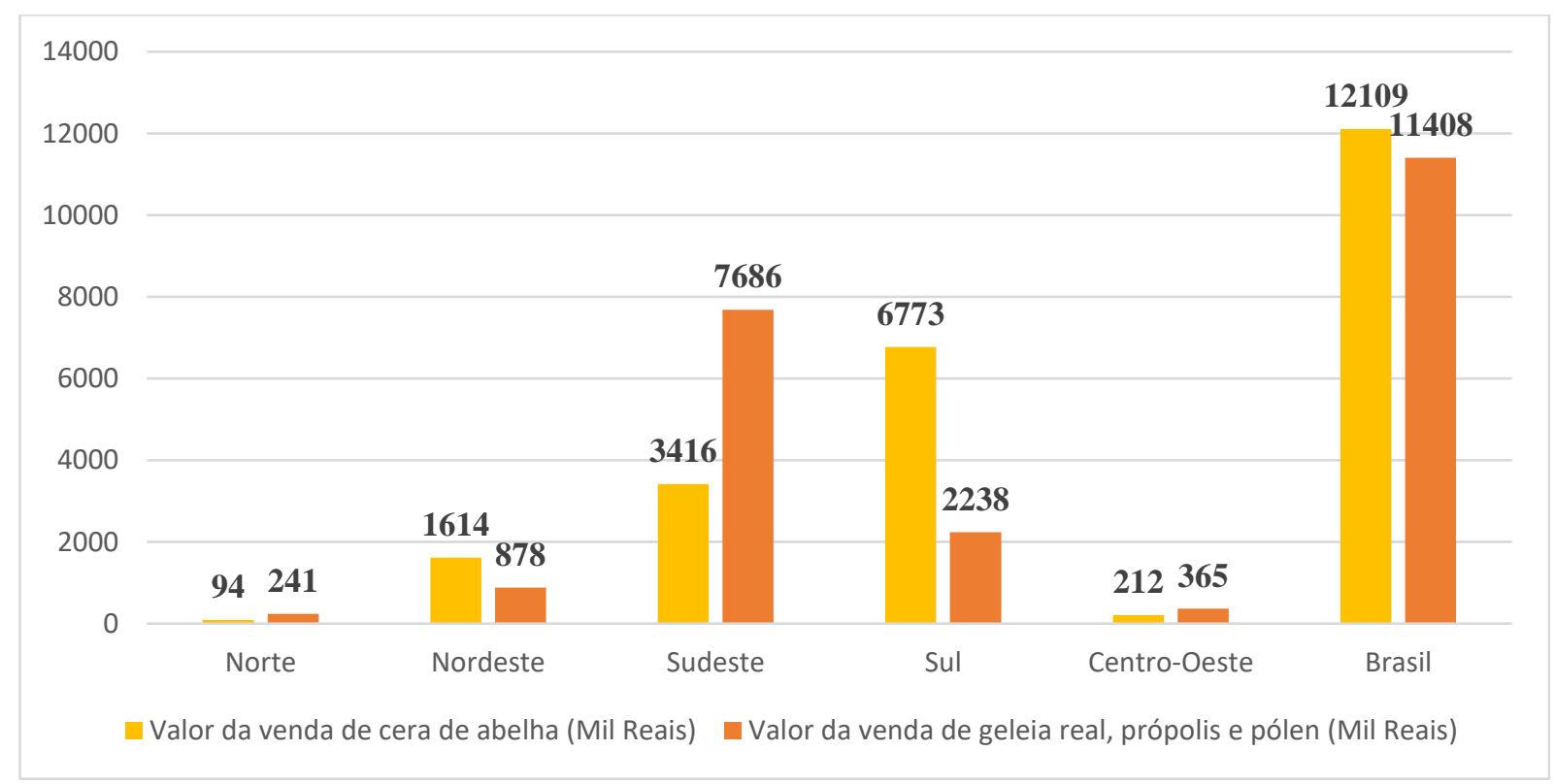

Fonte: IBGE (2017).

Nota-se um valor bem abaixo se comprado com a venda de mel, o destaque fica para região Sudeste como a região que mais comercializa produtos apícolas (cera de abelha, geleia real e própolis), em segundo lugar a região Sul e em terceiro a região Nordeste. 
Os canais para a comercialização de produtos apícolas ainda são limitados, ainda a problemas com a inserção das tecnologias e com a questão da gestão rudimentar (Dantas et al., 2020). Porém, é consenso entre os pesquisadores e apicultores de que há espaço para crescer, e isso pode acontecer a partir do momento que as cooperativas e associações entenderem que é imprescindível uma gestão profissionalizada, o que vai permitir que ocorra a prospecção de compradores de maior envergadura e, proporcionará consequentemente um espaço favorável para que os produtores invistam mais em gestão e tecnologia, aumentando sua produção e qualidade do mel (Vidal, 2020; Silva et al., 2020).

Outro aspecto importante que tem direta influência na comercialização é a inópia de um programa de marketing específico para a divulgação dos produtos apícolas. Na maioria dos casos essas ações são apenas pontuais, o que contribui para o baixo consumo de mel no Brasil (Nunes \& Heindrickson, 2019). Para aumentar a comercialização e o consumo é preciso um programa para divulgação dos produtos apícolas com ênfase na importância do mel enquanto alimento; na degustação de alimentos feitos com mel; na divulgação de produtos como cosméticos e aromatizantes de mel e entre outros. Essas campanhas devem ser direcionadas a diversos tipos de consumidores.

\section{Exportação de Produtos Apícolas}

Nunes e Heindrickson (2019) relatam que a cadeia produtiva da apicultura nacional atravessa um momento excepcional. O Brasil deu início a partir do ano 2000 um processo de inserção de produtos apícolas no mercado internacional, rapidamente alçando projeção como um país exportador. Dentre os produtores principais, se destacam três grupos: o primeiro com a China, cujo atual volume de produção é superior a 550 mil toneladas; o segundo com a presença da Argentina que produz anualmente acima de 70 mil toneladas; e em terceiro um conjunto de países, com produção entre 20 mil a 60 mil toneladas, onde se inclui o Brasil. Entre o ano de 2013 e 2017 a produção chinesa aumentou em cerca de 90 mil toneladas (Vidal, 2020).

Na tabela 2 mostra o número de toneladas exportadas dos principais países produtores de mel.

Tabela 2 - Número em mil tonelada de mel exportadas dos principais países produtores.

\begin{tabular}{llllll}
\hline Países & 2012 & 2013 & 2014 & 2015 & 2016 \\
\hline China & 112.962 & 128.654 & 134.820 & 150.972 & 131.649 \\
Argentina & 75.135 & 65.180 & 54.500 & 45.659 & 81.183 \\
Ucrânia & 13.338 & 21.674 & 36.336 & 36.013 & 48.605 \\
Vietnam & 13.200 & 34.924 & 49.559 & 39.823 & 42.265 \\
Índia & 24.515 & 30.099 & 26.976 & 40.829 & 35.793 \\
México & 32.040 & 33.458 & 39.152 & 42.161 & 29.098 \\
Espanha & 20.459 & 21.579 & 26.834 & 30.590 & 27.422 \\
Brasil & 16.707 & 16.181 & 25.317 & 22.206 & 24.203 \\
Alemanha & 21.109 & 20.885 & 22.667 & 23.732 & 23.795 \\
Bélgica & 16.557 & 20.144 & 20.007 & 25.897 & 20.816 \\
\hline
\end{tabular}

Fonte: FAOSTAT (2019).

A china além da maior produtora, também é o maior exportador mundial de mel. As exportações da China foram de 131.000 toneladas em 2016, ante 112.000 toneladas no ano de 2012. A Argentina vem posterior a China, tendo exportado 81.000 toneladas em 2016, quantidade superior à produção do país naquele ano possivelmente devido da venda de estoques não comercializados nos anos anteriores, quando as exportações do país haviam decrescido. A Ucrânia é o terceiro e o Brasil é o oitavo (24 mil toneladas em 2016, segundo a Comex foram exportadas cerca de 28 mil toneladas em 2017) (COMEX, 2019).

Quanto ao destino das exportações de mel brasileiro, o maior importador é os Estados Unidos, destino de quase $80 \%$ da produção exportada no ano de 2018, que foi de cerca de 23.000 mil toneladas (Vidal, 2020). Logo em seguida aparecem Alemanha, Canadá, Holanda Reino Unido como os principais destinos do mel exportado pelo Brasil. Apesar do aumento do 
volume exportado em 2018 comparado a 2017, os valores das exportações caíram de U\$ 121 milhões de dólares para U\$ 95 milhões, em decorrência da queda no preço do produto de U\$4,48/kg para U\$3,34/kg (Nunes \& Heindrickson, 2019).

Alguns países importam e depois exportam (caso da Alemanha), de maneira que as quantidades que importam não têm enquanto destino final seu mercado interno, pois claramente reexportam o mel (Santos et al., 2020). Em termos de importações, a Alemanha e Estados Unidos são os players com maior destaque, com respectivamente 21,3\% e 30,0\% do total. Os maiores exportadores respectivamente são China com 24,8\% e Argentina com 20,2\% (Vidal, 2020).

A cadeia produtiva do mel no Brasil é pouco organizada e simples em termos de mecanismos de negociação e controle com o mercado (Nunes e Heindrickson, 2019). Nos últimos anos surgiu a tentativa de instituir cadeias estruturadas com cooperativas e associações de apicultores, como também de traders, com o objetivo de assegurar qualidade aos produtos destinados ao exterior (Pinheiro, 2017). A partir da produção, extração e cultivo, a cadeia produtiva dos produtos apícolas, toma duas direções: o processamento e beneficiamento próprio, ou terceirizado em grandes empresas e Arranjos Produtivos Locais (Farias \& Miranda, 2016).

Um exemplo disso é quando o mel é processado nas "Casas de Mel" e os entrepostos que são responsáveis pelo processamento e podem ser vinculados às associações, instituídas por órgãos públicos ou produtores (Farias \& Miranda, 2015). Após esse processamento, o mel é enviado para os distribuidores que transportam esse produto até as cooperativas, supermercados, lojas especializadas e etc., chegando, por fim até o consumidor. Existe também a venda direta, realizada pelo produtor ou pelas associações e cooperativas (Cunha, Machado \& Costa, 2014).

Em tempos de migração e colheita, os produtores podem se unir e dividir custos. Transformar essa participação individual em comunitária constitui como uma "alavanca" para o crescimento da capacidade comercial e produtiva dos associados. Em associação, os produtores ganham força coletiva para dirigir os passos do desenvolvimento da comunidade (Farias \& Miranda, 2016).

No Gráfico 7 é possível analisar a participação das regiões nos valores das exportações de mel brasileiro em 2018.

Gráfico 7 - Representação da exportação de mel por estado da região Nordeste do Brasil entre 2011 e 2020.

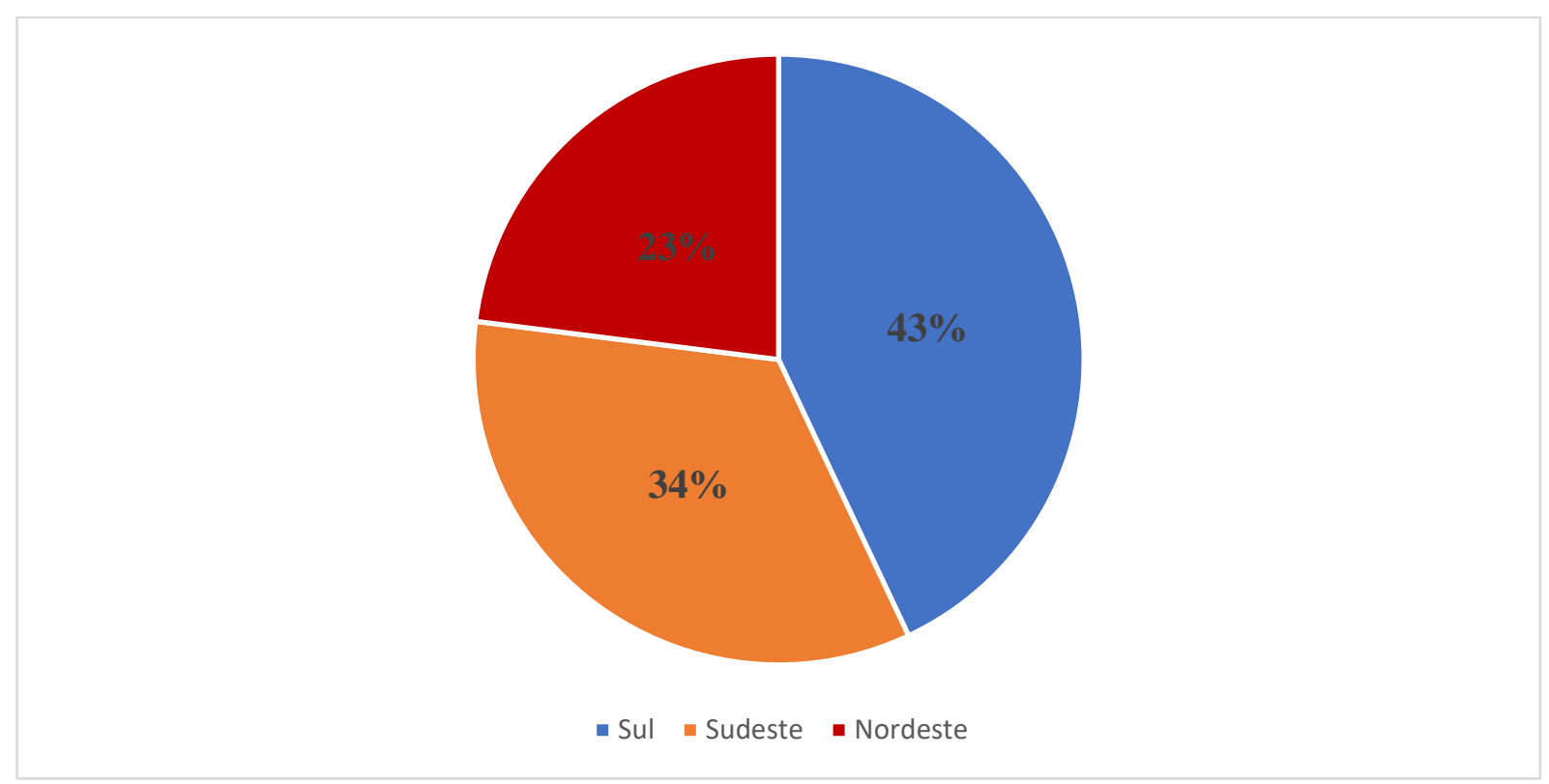

Fonte: MAPA (2020). 
Dentre as regiões exportadoras o Sul no ano de 2018 exportou US\$ 41.385.514, o que representou 43\% da exportação brasileira de mel naquele ano, em segundo lugar a região Sudeste com US\$32.331.942 e terceiro lugar a região Nordeste com US\$21.702.326.

As exportações brasileiras de mel se concentram em oito estados, com destaque para São Paulo com maior média, sendo o maior exportador nacional, com US\$20,56 milhões por ano, Santa Catarina U\$ 14,62 milhões por ano e Paraná U\$10,38 milhões por ano (Nunes \& Heindrickson, 2019). É importante mencionar que há empresas especializadas que compram o mel de produtores de outras regiões e exportam nesses estados, o que pode inflar os dados de exportação destes (Santos et al. 2020).

No primeiro bimestre de 2021, o Brasil exportou cerca de 9 mil toneladas de mel, registrando um aumento de mais de 100\% em relação ao mesmo bimestre de 2020. O Piaú foi o estado que mais contribuiu para esse aumento, com um volume de 2,8 mil toneladas exportadas do produto, sendo oriundas dos municípios de Oeiras (2,1 mil), Picos (325) e Simplício Mendes (38) (AGROSTAT, 2021).

$\mathrm{Na}$ cadeia nordestina apícola, coexistem vários canais de distribuição, desde os mais simplistas, em que o produtor vende diretamente o seu produto ao consumidor final, até aqueles mais modernos com a presença de vários intermediários (Vidal, 2020). A intermediação acontece por meio de agentes primários (associações de apicultores, entrepostos ou cooperativas), é exercida geralmente por um apicultor da localidade que se especializa na comercialização. Esses agentes podem comercializar com fracionadores/processadores, mercados varejista e atacadista e ainda vender o mel para o consumidor final. Porém, na maioria dos casos, o intermediário atua no canal de comercialização do mel a serviço de entrepostos, onde a sua remuneração ocorre das comissões sobre o volume de mel comercializado (Santos et al., 2020).

Analisando a exportação da região Nordeste, alguns Estados se destacam a nível nacional como é o caso de Bahia e Piauí, devido ao grande número de apicultores eles repassam a sua produção para as cooperativas, e estas a encaminham para uma cooperativa central, que vende a produção para as empresas exportadoras. No Piaú existe a própria Casa Apis (Central de Cooperativas) que exporta a produção (Vidal, 2020).

No Maranhão e Ceará muitos apicultores comercializam sua produção por meio dos intermediários devido à inexistência de uma estrutura sólida de alguma modalidade associativa organizacional que possa administrar o elo distributivo da produção (Liberato \& Morais, 2016).

O Rio Grande do Norte, Pernambuco, Sergipe e Alagoas de acordo com o Ministério da Agricultura, Pecuária e Abastecimento - MAPA (2020) ainda não possuem estabelecimentos habilitados para exportar produtos apícolas, assim, parte do volume do mel produzido nesses estados é comercializada para os representantes de empresas exportadoras dos estados vizinhos e estados do Sudeste do país.

A Paraíba obteve produção de mel de 157 toneladas em 2017 e ocupou a $5^{\text {a }}$ posição de maior produtor entre os estados da região Nordeste (IBGE, 2017). Porém, alguns entraves como a inópia de cursos profissionalizantes e não aplicabilidade dos padrões de qualidade, acabam por dificultar a venda do mel no mercado externo e interno (Silva et al., 2020; Dantas et al., 2020).

Vidal (2020) também aponta algumas dificuldades para a comercialização de produtos apícolas no Nordeste: o pequeno produtor, em muitos casos, não tem condições de transportar grandes quantidades e também tem dificuldades na questão do armazenamento, além de necessitar de terceiros para revender, certificar ou exportar o seu produto. Consequentemente, parte da produção é enviada ao intermediário, que adquire o mel para revenda nacional ou para exportação a um preço bem abaixo do mercado, ficando com a maior margem de lucro. 


\section{Considerações Finais}

O mercado nacional e internacional de produtos apícolas pode ser uma importante fonte econômica para os produtores, e uma oportunidade para o Brasil, uma vez que esse mercado apresenta demanda crescente e constante, nos últimos anos, tendo como resultado o crescimento das exportações.

O Brasil se apresenta destaque no mercado mundial, demostrado pela sua evolução na participação mundial, com taxas de crescimento constantes e expressivas. As exportações de produtos apícolas nordestinos são competitivas no mercado nacional e internacional. Embora a produção de produtos apícolas seja realizada por grande número de apicultores, verifica-se a existência de grandes empresas e indústrias atuando no setor e respondendo por grande parte da comercialização, ficando maior parte dos lucros.

No cenário mundial, é importante mencionar a forte concorrência de alguns players mundiais (China e Argentina), que ditam os preços nos mercados. Daí a necessidade de a região Nordeste assegurar a qualidade e uniformidade dos produtos, com a finalidade de conservar a parcela desse mercado conquistada nos últimos anos e buscar novos mercados na esfera internacional, sempre apoiada na vantagem comparativa dos produtos apícolas nordestino, em especial na sua qualidade e exoticidade.

Com relação ao mercado interno, a apicultura nos próximos anos precisa se movimentar para ampliar a participação no setor, o que até então é pouco explorada. A grande maioria da população ainda enxerga o mel não enquanto alimento, mas como um remédio, o que dificulta o estímulo ao consumo do produto.

Não obstante a grande gama de produtos que podem ser trabalhados na atividade moderna, o mel ainda é o principal produto apícola comercializado no Nordeste. Cabe destacar que a própolis, a cera e o pólen vêm ganhando mais espaço no mercado, principalmente pelas suas aplicações na introdução na dieta diária como alimento funcional.

Como sugestões para pesquisas futuras destacar importância de se fomentar a exploração e comercialização de outros produtos apícolas na região do Nordeste.

\section{Referências}

Araujo, J. L. P., Correia, R. C., \& Silva, E. M. S. da. (2016). Cadeia Produtiva do mel do território da borda do lago de Sobradinho, no Estado da Bahia. Revista SODEBRAS, 128(11), 96-101.

Aires-Neto, T. \& Carvalho, F. C. (2017). A meliponicultura tradicional no Rio Grande do Norte e arredores. In: Fonseca, V. L. I., Koedam, D. \& Hrncir, M. A abelha Jandaíra: no passado, presente e no futuro. Mossoró: EduFERSA, 109-114.

AGROSTAT. (2021). Estatísticas de Comercio Exterior do Agronegócio Brasil. Estatísticas de exportação e importação brasileira de produtos Apícolas. AGROSTAT. http://sistemasweb.agricultura.gov.br/pages/AGROSTAT.html.

Barbosa, E. S. \& Pereira, D. D. (2019). A apicultura no semiárido brasileiro. XV Semana de Agronomia, Centro de Ciências Agrárias, Universidade Federal da Paraíba. 07 a 10 de outubro.

Carvalho, L. X., Silva, W. B. De F., Carvalho, L. E. F., Lucena, F. C. \& Sales Junior, F. V. (2019). Apicultura e empoderamento: ressignificação do espaço de atuação da mulher na sociedade do alto oeste potiguar. Braz. J. of Develop., 5 (9), 14226-14245.

COMEX. (2019). Comex Stat. http://comexstat.mdic.gov.br/pt/home.

Cunha, A. C. C. de P., Machado, A. V., \& Costa, R. de O. (2014). Processamento, Conservação, Transporte e Comercialização do Mel no Brasil. Revista Brasileira de Agrotecnologia, 1(4), 24-29.

Dantas, M. C. A. M., Batista, J. L., Dantas, P. A. M., Dantas, I. M., Dias, V. H. P., Andrade Filho, F. C., Moreira, J. N., Mielezrski, G. L. N., Silva, M. G., Maia, A. G., Medeiros, A. C. \& Maracajá, P. B. (2020) Abelha sem ferrão e seu potencial socioeconômico nos Estados da Paraíba e Rio Grande do Norte. Research, Society and Development, 9(10), 1-37.

FAO. (2019). Food and Agriculture Organization of the United Nations. Faostat. http://www.fao.org/faostat/en/\#data.

Farfan, S. J., Celentano, D. Loch, V., Hernández-García, L., Silva-Junior C., \& Farfan, N. (2020). O bem comum na coprodução humana com a abelha nativa tiúba, Melipona fasciculata, na Baixada Maranhense. Anais do XI Congresso Brasileiro de Agroecologia, São Cristóvão, Sergipe, 15(2).

Farias, A. A., Sousa, F. A. S., Aires, T. L. B. A. \& Brito, T. C. (2021). A seca de 2012-2014 no município de Taperoá-PB e o papel das ações de convivência nesse contexto. Revista Brasileira de Geografia Física, 14(1), 135-158. 
Farias, J. S., \& Miranda, L. M. S. (2016). Ações Coletivas para a promoção de exportações do setor apícola brasileiro: O caso da associação Abemel. E\&G Economia e Gestão, 42(16), 116-137.

Gil, A. C. (2019). Métodos e Técnicas de Pesquisa Social. (7a ed.), Atlas.

Gomes, R. V. R. S., Miranda, M. E., Gomes, E. N., Sombra, D. S. \& Silva, J. B. A. (2017). Produção e qualidade de mel na zona da mata de Pernambuco. Centro Científico Conhecer, 14(26), 539-549.

IBGE. (2021). Instituto Brasileiro de Geografia e Estatística. Censo Agropecuário: SIDRA. https://sidra.ibge.gov.br/tabela/6935.

IBGE. (2017). Instituto Brasileiro de Geografia e Estatística. Pesquisa pecuária municipal. https://sidra.ibge.gov.br/Tabela/74.

Liberato, M. D. C. T. C., \& Morais, S. M. (2016) Produtos Apícolas do Ceará e Suas Origens Florais: Características físicas, químicas e funcionais. EdUECE, $179 \mathrm{p}$.

Marconi, M. A, \& Lakatos, E. M. (2017). Técnicas de Pesquisa. São Paulo: Atlas.

MAPA. (2020). Ministério da Agricultura, Pecuária e Abastecimento. Relação de Produtos Autorizados para os Estabelecimentos Brasileiros Exportarem por País. Departamento de Inspeção de Produtos de Origem Animal - DIPOA. http://bi.agricultura.gov.br/reports/rwservlet?sigsif_cons\&prod_aut_estab_ bra_exp_pais.rdf\&p_id_pais=\&p_id_mercado_comum=\&p_id_area=5\&p_id_produto=\&p_serial=1349412235\&paramform=no.

Nunes, S. P., \& Heindrickson, M. (2019). A cadeia produtiva do mel no Brasil: análise a partir do sudoeste Paranaense. Braz. J. of Develop., 9(5), 16950-16967.

Paula, M. F., \& Santos, A. J., Timofeiczyk Junior, R., Hoeflich, V. A., Silva, J. C. G. L., \& Angelo, H. (2016). Análise da competitividade das exportações brasileiras de mel natural, segundo o modelo constant market share e o índice de vantagem comparativa revelada. Rev. Ceres, Viçosa, 63(5), 614-620.

Pereira, D. S., Nakasone, A. K., Oliveira, L. C., Oliveira, M. S., Pereira, N. S., Cruz, J. N., Ports, P. S., Souza Filho, A. P. S., Medeiros, A. C., Silva, R. A., Maracajá, P. B., Freitas, M. O., \& Freitas, C. I. A. (2020). Efeito de extratos de própolis apícola amazônica sobre Xanthomonas axonopodis pv. passiflorae no Estado do Pará-Brasil. Research, Society and Development, 9(11), 1-16.

Pereira, R. M., Alves, T. T. L., Silva, J. N., Silva, I. M., Alencar, S. E. H., Souza, A. A., \& Silva, M. B. (2017). Perspectivas e desafios do arranjo produtivo local (APL) da apicultura no Município de Ouricuri, Estado Pernambuco. Revista Semiárido de Visu, 5(1), 30-37.

Pinheiro, S. E. B. (2017). Apicultura na região do Pampa Gaúcho: Caracterização do Mel e da cadeia Produtiva. Tese de Doutorado: Programa de Pós-Graduação em Engenharia Agrícola, Universidade Federal de Santa Maria - UFSM.

Santos, S. B., Mikos, A., Silva, E. A., Lopes, F. S. do C., Ribeiro, S. B., \& Jacobsen, R. H. F. (2020). Cadeia produtiva do mel: Agroindústria familiar em Rolim de Moura, Rondônia. Rev. Bras.de Gestão Ambiental, 3(14), 334-340.

Scaramucci, C. P., \& Silva, T. F. (2014). Cadeia Produtiva do Mel: Revisão de Literatura. Anais XVII Simpósio de Ciências Aplicadas da FAEF: Editora FAEF, 2014.

SEBRAE. (2009). Serviço Brasileiro de Apoio às Micro e Pequenas Empresas. Manual de segurança e qualidade para apicultura. Brasília, SEBRAE /NA.

SENAR. (2010). Serviço Nacional de Aprendizagem Rural. Mel: manejo de apiário para produção do mel. (2a ed.), SENAR.

SILVA, L. (2014). Por uma leitura sociotécnica da história da criação de abelhas no Brasil: análise à luz da Social Construction of Technology (SCOT). Mosaico Social - Revista do Curso de Ciências Sociais da UFSC, 12 (07).

Silva, M. G., Dantas, M. C. A. M., Moreira, J. N., Ferreira Neto, J., Pereira Junior, E. B., \& Rolim, H. O. (2018). Teor de umidade e potencial hidrogeniônico do mel de abelha Jandaíra produzido em Sousa, Paraíba. Revista Brasileira de Gestão Ambiental, 12(4), 07-12.

Silva, M. G., Santana, A. G., Silva, P. R. M., Silva, R. A., Medeiros, A. C., \& Maracajá, P. B. (2020). Rotulagem dos méis de Apis mellifera comercializados no Alto Sertão da Paraíba. ACTA Apicola Brasilica, 8, e7777.

Silva, E. A. da. (2010). Apicultura Sustentável: Produção e comercialização de mel no sertão sergipano. Dissertação de Mestrado: Pós-Graduação em Desenvolvimento e Meio Ambiente, Universidade Federal de Sergipe.

Souza, B. A., Lopes, M. T. R., \& Pereira, F. M. (2012). Cultural aspects of meliponiculture, In: VIT, P; ROUBIK, D. W. (Eds). Stingless bees process honey and pollen in cerúmen pots, SABER-ULA, Universidad de Los Andes, Mérida, 1-6.

Souza, C. O., Teixeira, V. W., Teixeira, A. A. C., Cruz, G. S., Guedes, C. A., Nascimento, J. C. S., \& Teixeira, C. S. (2021). Consequências do uso excessivo de defensivos agrícolas em abelhas: Uma das prováveis causas do CCD.In; Meio ambiente: enfoque socioambiental e interdisciplinar / Organizadores Juliana Thaisa Rodrigues Pacheco, Mauricio Zadra Pacheco. Atena.

USDA. (2019). United States Department Of Agriculture. National honey report. www.marketnews.usda.gov/mnp/fv-home.

Vidal, F. (2019). Comércio Exterior do Agronegócio do Nordeste: Produtos Apícolas. Caderno Setorial ETENE, 4(77).

Vidal, F. (2020). Evolução da produção de mel na área de atuação do BNB. Caderno Setorial ETENE, 5(122). 